\title{
Conserved expression of transposon-derived non-coding transcripts in primate stem cells
}

\author{
LeeAnn Ramsay ${ }^{1}$, Maria C. Marchetto ${ }^{2}$, Maxime Caron ${ }^{1,3}$, Shu-Huang Chen ${ }^{3}$, Stephan Busche ${ }^{3}$, \\ Tony Kwan ${ }^{1,3}$, Tomi Pastinen ${ }^{1,3}$, Fred H. Gage ${ }^{2}$ and Guillaume Bourque ${ }^{1,3^{*}}$ (D)
}

\begin{abstract}
Background: A significant portion of expressed non-coding RNAs in human cells is derived from transposable elements (TEs). Moreover, it has been shown that various long non-coding RNAs (IncRNAs), which come from the human endogenous retrovirus subfamily $\mathrm{H}(\mathrm{HERVH})$, are not only expressed but required for pluripotency in human embryonic stem cells (hESCs).

Results: To identify additional TE-derived functional non-coding transcripts, we generated RNA-seq data from induced pluripotent stem cells (iPSCs) of four primate species (human, chimpanzee, gorilla, and rhesus) and searched for transcripts whose expression was conserved. We observed that about 30\% of TE instances expressed in human iPSCs had orthologous TE instances that were also expressed in chimpanzee and gorilla. Notably, our analysis revealed a number of repeat families with highly conserved expression profiles including HERVH but also MER53, which is known to be the source of a placental-specific family of microRNAs (miRNAs). We also identified a number of repeat families from all classes of TEs, including MLT1-type and Tigger families, that contributed a significant amount of sequence to primate IncRNAs whose expression was conserved.
\end{abstract}

Conclusions: Together, these results describe TE families and TE-derived IncRNAs whose conserved expression patterns can be used to identify what are likely functional TE-derived non-coding transcripts in primate iPSCs.

Keywords: Transposable elements, Long non-coding RNAs, Induced pluripotent stem cells

\section{Background}

It has been shown that the majority of the human genome is transcribed but that most of the resulting RNA products do not encode for proteins $[1,2]$. Notably, some of the long non-coding RNAs (lncRNAs), defined as non-coding transcripts longer than 200 base pairs, are known to play important biological roles [3-5]. Moreover, it has been shown that an important source of lncRNA sequences are transposable elements (TEs), which make up about $50 \%$ of the human genome [6]. Specifically, it was reported that many lncRNAs are initiated in TEs and that about $75 \%$ of them have at least one exon overlapping a TE [7]. Actually, it has also been proposed that TE-derived sequences

*Correspondence: guil.bourque@mcgill.ca

'Department of Human Genetics, McGill University, 1205 Dr Penfield Avenue, H3A 1B1 Montreal, Canada

${ }^{3}$ McGill University and Genome Quebec Innovation Centre, 740 Dr Penfield Avenue, H3A 1 A4 Montreal, Canada

Full list of author information is available at the end of the article in lncRNAs may provide pre-formed functions to these transcripts $[7,8]$.

One example of a TE-derived lncRNA is lnc-RoR, a transcript implicated in the modulation of reprogramming of human iPSCs [9], which initiates in the human endogenous retrovirus subtype $\mathrm{H}$ (HERVH) [7]. HERVH is one of the most abundant human endogenous retroviral families in the human genome with about 1000 copies [10] and recent studies have found that HERVH instances are highly and specifically expressed in human embryonic stem cells (hESCs) [11, 12]. Moreover, it was shown that the expression of these TE-derived lncRNA transcripts helps define the naive stem-cell state [13] and knockdown experiments confirmed that this expression is essential for the maintenance of pluripotency in human stem cells [14]. HERVH-derived lncRNAs are probably not the only TEderived transcripts involved in stem cell pluripotency, as knockdowns of several lncRNAs result in exit from the pluripotent state [15]. 
TE sequences are repeated throughout the genome because of their ability to replicate and insert into genomic DNA. There are several mechanisms through which this replication can occur, which defines the broadest classification of TEs: DNA, ERV/LTR, LINE and SINE [16]. TEs have freqently been ignored in genomic studies because of their repetitive nature, which makes them more difficult to deal with computationally, but their impact has gained recognition as many of them have been shown to be involved in the formation of new transcripts $[13,14]$ and regulatory innovations [17-19]. Although some TEs have a well characterized function in their host, such as in pluripotency [14] and X-chromosome dosage compensation [20], the majority of them have no known function. Comparative genomic studies have been shown to be a powerful way to identify functional elements in the genome [21]. An early study of this type looking at TEs noted that conserved repeats were preferentially located near genes that were associated with development and transcription regulation [22]. More recent studies focusing on lncRNAs found that these transcripts were expressed in a highly tissue specific manner [23] and even more so than protein coding genes [24]. Research examining cross-species lncRNA expression found that this high level of tissue specificity was well conserved in primates, but not in more distant species [23]; and that about 30\% of lncRNAs were primate specific [25].

While a number of studies have examined the expression and evolution of non-coding RNAs in mammals $[26,27]$, none have focused on primate non-coding RNAs and on the link between TEs and lncRNAs. To identify TE-derived non-coding RNAs with important genomic functions, such as HERVH, we posited that cross-species expression data would be informative. In this context, and because of the rapid evolution of the lncRNA repertoire, we generated RNA-seq data from induced pluripotent stem cells (iPSCs) of several primate species: human (Homo sapiens), chimpanzee (Pan troglodytes), gorilla (Gorilla gorilla gorilla), and rhesus (Macaca mulatta). Using this resource, we looked for TE-derived non-coding transcripts with conserved expression profiles.

Using the same RNA-seq data, we also developed an iPSC-specific lncRNA catalogue for human, chimpanzee, gorilla, and rhesus. With this catalogue, we were able to identify repeat families that have contributed the most DNA to primate iPSCs lncRNAs. We were also able to identify several TE-derived lncRNAs, such as HERVH, that are well conserved in terms of having a large number of orthologous instances that are expressed in human and in some of the non-human primate (NHP) species. Several of these well conserved TE-derived lncRNAs have not been characterized before and could be novel functional transcripts.

\section{Results}

\section{Conservation of TE instances in primate genomes}

We first wanted to examine the conservation of TE instances between human and NHP species before looking at any expression data. By conservation here we mean TE instances occurring at orthologous locations in the different primate species and not having been lost in one of the genomes. To do so, we performed various pairwise comparisons to determine how many TEs occur in corresponding genomic locations. First, TE annotations were generated for human, chimpanzee, gorilla, and rhesus using the RepeatMasker software [28] (Methods). As expected, the repeat catalogues were found to be comparable in size, with each species having between 4.2-4.5 million TE instances (Additional file 1: Table S1). Next, conserved TEs were identified by determining if they existed at orthologous genomic locations using the UCSC LiftOver tool [29]. TEs were labeled as putatively conserved if they could successfully LiftOver between species (Methods). We restricted the analysis to TEs which did not overlap coding regions, as we are interested in the contribution of TEs to non-coding transcripts.

Overall we found that more than $90 \%$ of repeat instances across all major repeat families were conserved between human and chimpanzee or gorilla, about $85 \%$ were conserved in rhesus and over $80 \%$ were conserved across all analyzed primate species (Fig. 1a). This high conservation between human and NHPs can be explained by their relatively recent divergence. We note that repetitive sequences are sometimes missing from assemblies of lower quality, such as the ones we are using here for the NHP, and so these estimates should be taken as lower bounds.

We can verify our approach to identify conserved TE instances by using the well-characterized HERVH repeat family. Using the above annotation strategy, human, chimpanzee, and gorilla were found to have 1266, 1157, and 1276 HERVH instances, respectively. This is consistent with recent estimates that indicated that approximately 1000 insertions occurred in higher primates [30]. Rhesus has fewer HERVH instances (742), which can by explained by the fact that this family expanded after the divergence from rhesus. We can also examine the regions of the HERVH consensus sequence that are being attributed to these insertions (Additional file 1: Figure S1). The patterns observed are consistent with the known evolutionary history of HERVH, with a large expansion that took place after the split from rhesus and that contain a deletion in what used to correspond to the ENV gene [31]. To verify that LiftOver is identifying orthologous sequences accurately, we examined the TE annotation of conserved human HERVH instances in NHPs. We found that of the human HERVH instances that successfully lift to chimpanzee and gorilla over $90 \%$ were independently annotated as HERVH in that species (Additional file 1: 

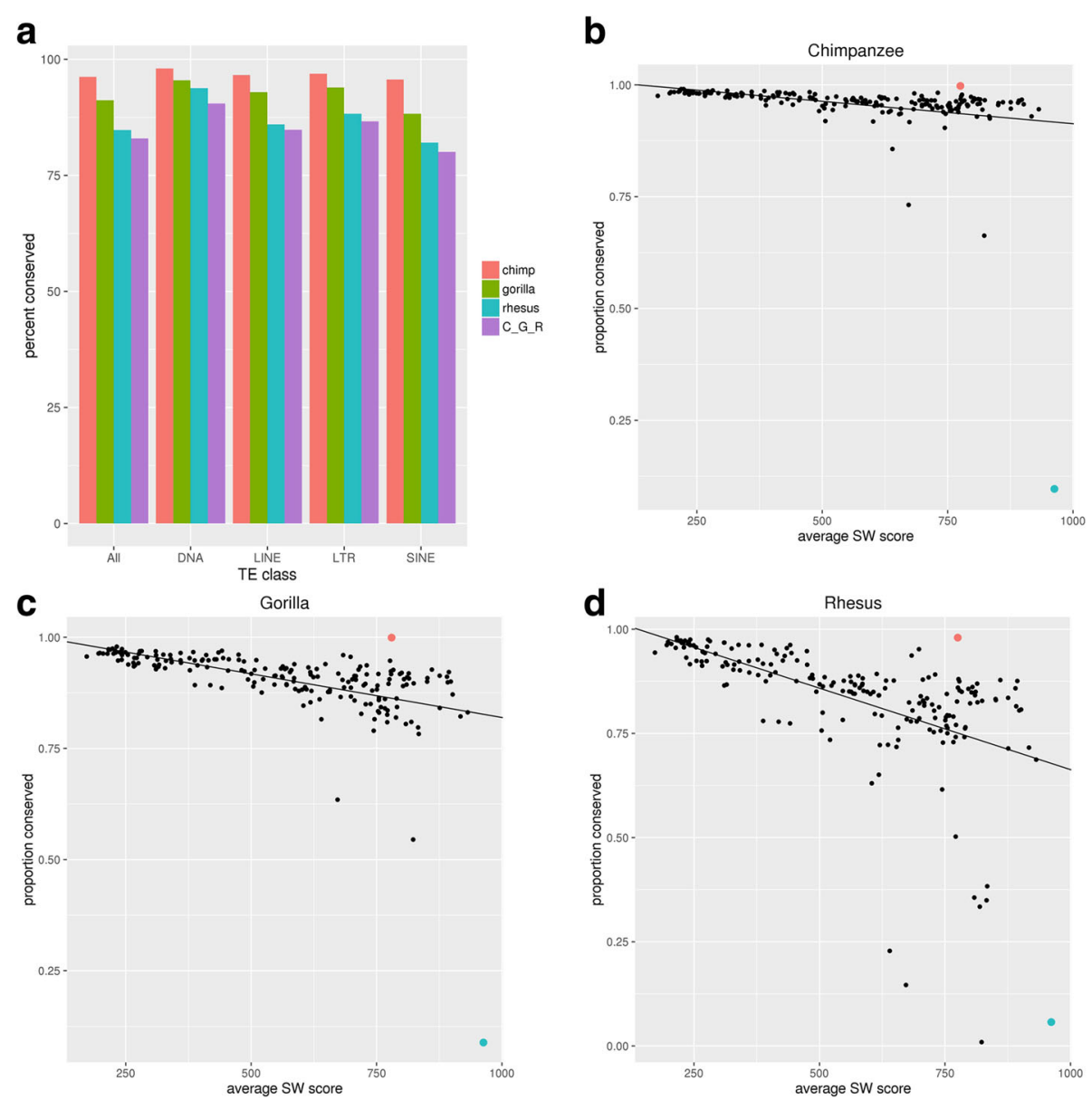

Fig. 1 a Percent of all human TEs which are conserved in NHP grouped by TE class. C_G_R indicates TEs conserved in all 3 NHP species. b The proportion of instances in each TE family that are conserved in Chimpanzee ( $y$-axis) relative to the average sequence identity score of the family (X-axis). Sequence similarity to the family's consensus sequence is shown as a scaled Smith-Waterman (SW) score. This is used as a surrogate for the age of the TE family. Older TEs are on the left, and newer insertions are to the right. TE families which inserted more recently are less conserved than older TEs. TE families were filtered for those with more than 30 expressed instances in human. tRNA-Asn-ACC in red, AluYa5 in blue. c The same in Gorilla (d) The same in Rhesus

Table S2), which further supports the accuracy of the methodology.

Next, we were interested in TE conservation at the level of families. Using a method previously described to estimate the age of each family [32] (Methods), we observed that the older TE families were more conserved than recent ones (Fig. 1b-d and Additional file 2: Table S3). This is to be expected given that the younger families tend to be associated with more recent expansions whose instances can be absent from the other genomes. This downward trend was most prominent in rhesus, the most distant analyzed species. In this analysis, one outlier TE family appears to be conserved more than expected based on its family age (shown in red in Fig. 1b-d). This TE family is tRNA-Asn-AAC, a repetitive non-coding gene which produces a tRNA. It is classified as a TE due to its repetitive nature, but because it is a functional tRNA conserved across all mammalian cells, its high level of conservation can be explained. This TE family has 46 instances in the human genome, not overlapping annotated coding regions, 45 of which are conserved in the NHPs we studied. In contrast, AluYa5 (shown in blue in Fig. 1b-d) is a very recent family which is poorly conserved between human and rhesus. The AluY family is still active in the human genome [33]. AluYa5 has 2290 instances not overlapping coding regions, only 131 (5.7\%) of which are annotated as putatively conserved in rhesus.

\section{TE expression is conserved between human and non-human primates}

Next, we wanted to examine TE instances with conserved expression profiles in primate iPSCs. We generated RNAseq data from four primate iPSC cell lines: 3 human, 1 chimpanzee, 2 gorilla, and 1 rhesus (Methods and 
Additional file 1: Table S4). We found that TE instances that had an expression level of at least 1 RPKM in human and did not overlap protein coding genes were conserved, in terms of having an orthologous locus in NHP, at slightly lower levels than TEs in general (Fig. 2a). This is probably because expressed TEs tend to be younger than nonexpressed TEs (Additional file 1: Figure S2). Still, over 70\% of expressed human TEs were found to have an orthologous sequence in all NHP species studied. Next, when we looked to see which of these conserved TE instances were also expressed in the other species, we observed that about $33 \%$ of them had orthologous expression in chimpanzee and slightly less in gorilla (Fig. 2b). This type of expression conservation dropped dramatically when rhesus was taken into account. Clearly although many TE instances have been retained in both human and rhesus, they are also under different expression controls in the two species. In this context, TEs that do exhibit expression conservation between human and rhesus are especially interesting since expression has been conserved over a long evolutionary time period.

To further examine the statistical significance of the conservation of expression at the level of individual repeat families, we performed a hypergeometric test (Methods). We removed small repeat families, Simple Repeats, and low complexity repeats from this analysis since they are more prone to biases [32]. Looking at expression conservation based on the level of sequence conservation of the family did not reveal any unusual patterns (Additional file 1: Figure S3 and Additional file 2: Table S3). The TE families that had the highest proportion of instances with conserved expression between human and chimpanzee are shown in Table 1 (see also Additional file 3: Table S5). In particular, the MER53 repeat family had the largest percentage of TEs with such conserved expression $(51 \%$, $p$-value $=9.61 * 10^{-27}$, Fig. 2c). Notably, this TE family
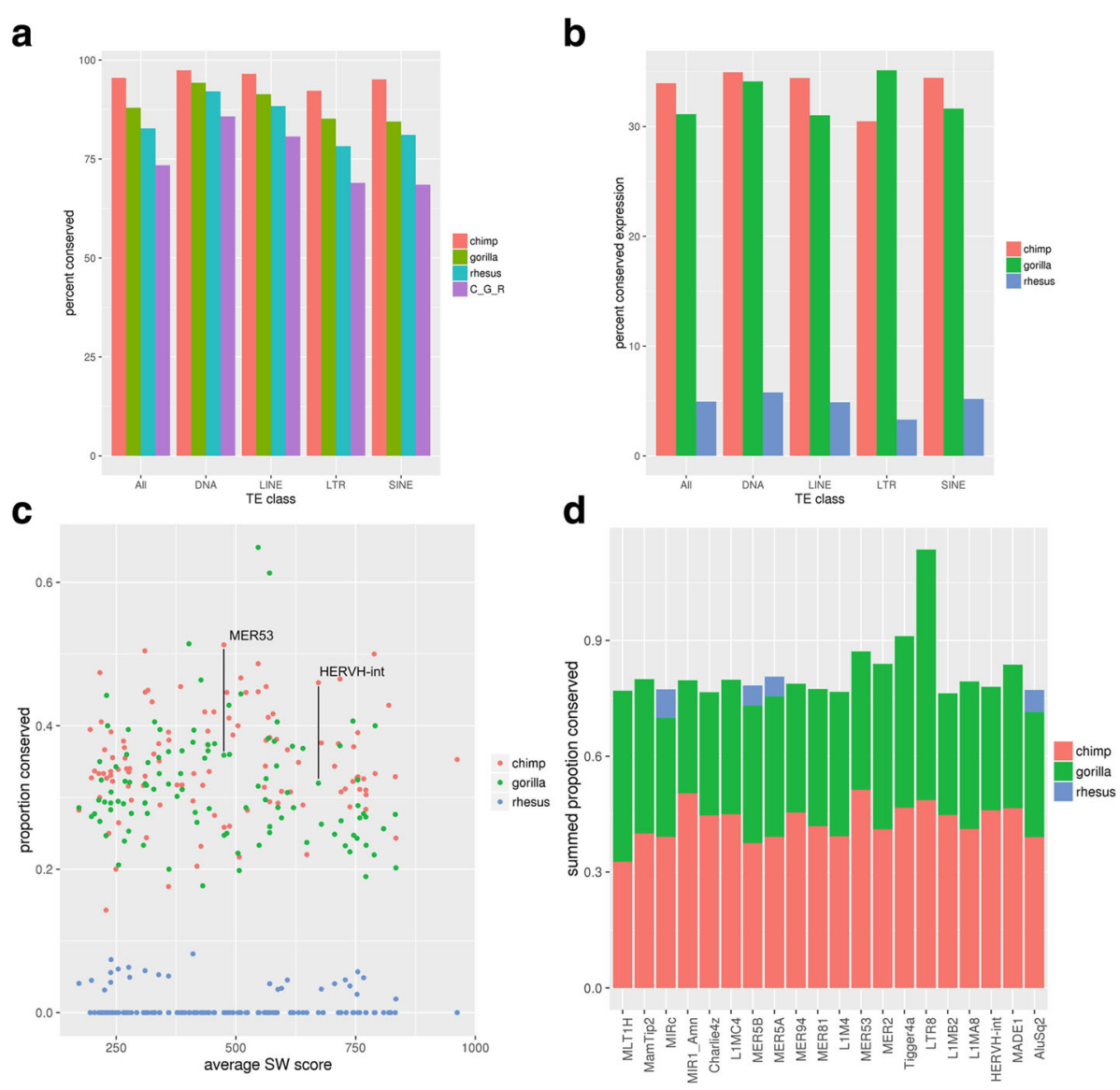

Fig. 2 a Percent of all TEs expressed in human which are conserved in NHPs, grouped by class. C_G_R indicates TEs conserved in all 3 NHP species. b Percent of human expressed TEs which are conserved in terms of sequence and expressed in NHPs. c TE families plotted by average sequence identity and proportion conserved in NHPs. Y axis specifies the proportion of human expressed TEs which are also expressed in NHPs. Average sequence divergence is used as a surrogate for age, with older TEs on the left. This plot shows only families with 30 or more expressed TEs and 10 or more conserved in the target species. d Top 20 most conserved families (sequence and expression) when their conservation is summed across all 3 NHP species 
has previously been shown to be the source of a placentalspecific family of miRNAs [34], the miR-1302 family, and to be expressed in human embryonic stem cells [35]. The instances of MER53 that are highly expressed and conserved among primates do not overlap with annotated miR-1302 transcripts, however the annotated transcripts are only ones that have been experimentally validated. The fact that the expression of the MER53 instances is highly conserved suggest a potential role for this family in both human and chimpanzee iPSCs.

Another well characterized example that appears in Table 1 is HERVH with 50 instances expressed in human, which is comparable to what was found in previous studies $[11,12,14]$. Notably, we found 23 orthologous instances also expressed in chimpanzee, which makes it one of the families with the highest expression conservation $\left(46 \%, p\right.$-value $\left.=9.26 * 10^{-15}\right)$. The combined expression conservation of human expressed TEs in NHP also revealed HERVH as one of the top conserved TE families (Fig. 2c-d). We observed similar numbers of HERVH instances expressed in human, chimpanzee, and gorilla (around 5\%) and very few instance expressed in rhesus (Additional file 1: Figure S4). The differences we observe between human, chimpanzee, and gorilla HERVH expression are fairly small considering the size of the family and the number of instances expressed, which are both fairly high compared to most TE families. The low number of expressed HERVH in rhesus is expected since rhesus has fewer copies of this repeat family. The high conservation of expression of HERVH suggest that the HERVHlncRNA function revealed in human $[13,14]$ arose before the divergence of gorilla.

Finally, it has been noted that some Alu repeats exhibits mobilization in iPSCs [36] and a few Alu families are found to have high expression conservation (Table 1). However, most of the other TEs on this list have limited literature describing them, so further validation would be needed to determine if they play a role in primate iPSCs.

\section{iPSC-specific IncRNAs in primates frequently overlap transposable elements}

Several studies have shown that a large portion of lncRNA sequence is made up of TEs $[7,24]$. Since we were able to identify a number of TE families with significant conservation of expression between primate species we were interested to see if some of these TEs also contributed

Table 1 TE expression conservation between human and chimpanzee iPSCS

\begin{tabular}{|c|c|c|c|c|c|c|}
\hline Family & Class & Total & $\begin{array}{l}\text { Human } \\
\text { expressed }\end{array}$ & $\begin{array}{l}\text { Chimpanzee } \\
\text { expressed }\end{array}$ & Proportion & $p$-value \\
\hline MER53 & DNA & 5308 & 39 & 20 & 0.51 & $9.61 * 10^{-27}$ \\
\hline MIR1_Amn & SINE & 9495 & 113 & 57 & 0.50 & $1.71 * 10^{-71}$ \\
\hline AluSg7 & SINE & 5780 & 50 & 25 & 0.50 & $6.81 * 10^{-35}$ \\
\hline LTR8 & LTR & 2516 & 37 & 18 & 0.49 & $1.81 * 10^{-25}$ \\
\hline L2d & LINE & 19063 & 135 & 64 & 0.47 & $2.32 * 10^{-84}$ \\
\hline Tigger4a & DNA & 3242 & 45 & 21 & 0.47 & $2.41 * 10^{-20}$ \\
\hline MADE 1 & DNA & 7634 & 86 & 40 & 0.47 & $7.59 * 10^{-51}$ \\
\hline HERVH-int & LTR & 1266 & 50 & 23 & 0.46 & $9.26 * 10^{-15}$ \\
\hline MER94 & DNA & 4884 & 33 & 15 & 0.45 & $2.17 * 10^{-21}$ \\
\hline L1MC1 & LINE & 7375 & 33 & 15 & 0.45 & $8.19 * 10^{-22}$ \\
\hline L1MC4 & LINE & 12920 & 109 & 49 & 0.45 & $4.17 * 10^{-64}$ \\
\hline L1MB2 & LINE & 4967 & 38 & 17 & 0.45 & $1.49 * 10^{-20}$ \\
\hline Charlie4z & DNA & 5255 & 47 & 21 & 0.45 & $1.38 * 10^{-27}$ \\
\hline L1MB8 & LINE & 9006 & 56 & 25 & 0.45 & $7.32 * 10^{-34}$ \\
\hline L1M2 & LINE & 6281 & 56 & 25 & 0.45 & $4.05 * 10^{-32}$ \\
\hline OldhAT1 & DNA & 1897 & 30 & 13 & 0.43 & $5.22 * 10^{-16}$ \\
\hline AluYk3 & SINE & 5421 & 42 & 18 & 0.43 & $5.12 * 10^{-24}$ \\
\hline MER81 & DNA & 3551 & 31 & 13 & 0.42 & $4.35 * 10^{-18}$ \\
\hline L1MC3 & LINE & 6596 & 31 & 13 & 0.42 & $1.36 * 10^{-17}$ \\
\hline MER1B & DNA & 5060 & 36 & 15 & 0.42 & $2.05 * 10^{-20}$ \\
\hline
\end{tabular}

Here we examine only large families ( $\geq 100)$ from the main repeat classes (DNA, SINE, LINE, LTR). The table is sorted by the proportion of human expressed TEs which are conserved in chimpanzee. Only repeat families with at least 30 expressed instances in human are shown 
to lncRNA transcripts with conserved expression. Publicly available lncRNA catalogues for non-human primates are not nearly as complete as the human IncRNA catalogues. For this reason we created iPSC-specific IncRNA annotations for each species based on our iPSC RNAseq data using the FEELnc pipeline [37] (Additional file 1: Figure S5, Methods and Additional file 1: Table S6). Briefly, using the transcripts assembled from the RNAseq data, we filtered the transcriptome for protein coding genes, mono-exonic transcripts, and any transcripts with protein coding potential. We chose to remove monoexonic transcripts from this analysis because it was not possible to avoid systematic false positives in that category without extensive manual curation.

We validated this automated method for annotating lncRNAs using RNA-seq data by comparing it against GENCODE. Our human annotation contains 9,332 lncRNAs, $90.8 \%$ of which overlapped the GENCODE catalog (green values in Fig. 3a). After performing expression analysis on GENCODE lncRNAs with our iPSC data we noted that about half of the iPSC expressed GENCODE lncRNAs did not appear in our annotation (data not shown). However, the majority of these missing transcripts are either overlapping protein coding genes or are mono-exonic transcripts, which is consistent with our selection criteria.

The FEELnc method for identifying lncRNAs that uses gene annotation as a guide for transcriptome assembly with Cufflinks will perform differently depending on the quality of that annotation. The smaller size of our lncRNAs catalogs in NHP (Additional file 1: Table S6) is likely due to having fewer replicates but also a reflection of the more limited annotation in those species. When we applied the FEELnc method without such guide annotation we obtained approximately the same number of lncRNAs in human, chimpanzee, and gorilla (Additional file 1: Table S6 and blue values in Fig. 3a). Such de novo lncRNA annotation for rhesus resulted in very few lncRNAs detected. This seems to be due to a number of factors including: poor genome assembly and fewer aligned reads for rhesus. Since removing the guide annotation during the transcriptome assembly reduces the number of human lncRNA transcripts to a number comparable to chimpanzee and gorilla, we suspect the quality of the annotation to be the main cause of discrepancy in the lncRNA catalogue size for the different primate species.

Finally, for each lncRNA catalogue, we examined the proportion of the lncRNAs which had at least $10 \%$ of one exon overlapping a TE (Methods). In human we found that about $73 \%$ of the IncRNAs overlapped a TE (Additional file 1: Table S6), which is consistent with previous studies which examined human lncRNA catalogs [7, 24]. We found this proportion to be fairly consistent across all primate species, and also in the de novo lncRNAs cataglogues.

\section{Some TE-derived IncRNAs have conserved expression}

Finally, we were interested in the expression conservation of the primate iPSC IncRNAs. This analysis was done in conjunction with the TE annotations to identify TEderived lncRNAs whose expression is conserved. Using LiftOver to convert human lncRNA coordinates to NHP, we found that the majority of IncRNAs were conserved between human and NHPs (Table 2). For instance, of the 9,332 iPSC-specific lncRNAs in human, 7,226 have orthologous positions in chimpanzee. Next, we looked at which of these conserved lncRNAs were also expressed in the other species (Methods and Table 2). We found, in chimpanzee, that of the human IncRNAs that LiftOver, $40 \%$ were also expressed in chimpanzee.

Next, we labeled each lncRNA transcript with the TE it overlapped the most, whenever such an overlap corresponded to at least $10 \%$ of one of its exons (Methods). With such an annotation, we found no difference in the proportion of TEs contributing to all human lncRNAs and human lncRNAs with an orthologous NHP lncRNAs (Additional file 1: Figure S6). In the human lncRNAs, we observe a clear enrichment of LTRs and LINEs and a depletion of SINE elements as compared to the genomic proportion of these TEs (Fig. 3b). These patterns of enrichment and depletion of TE classes in lncRNAs are consistent with what has been observed in other studies $[7,24]$. Notably, we are now also able to observe the same patterns in NHP lncRNA catalogues (Fig. 3b).

When we investigated which TE families contributed the most sequence to lncRNA transcripts in human we noted that HERVH was one of the top contributors from the LTR class (Additional file 1: Figure S7). We also found that MLT1J was the member of the LTR family which contributed the most absolute sequence to human lncRNAs. Previous studies have shown that MLT1J harbors transcription factor binding sites for YY1 [38] and YY1 is known for repressing and activating a number of different promoters. In this way, the transposon MLT1J may have contributed YY1 binding sites to the genome, like the OCT4 binding sites in the promoter sequences of HERVH [18], thus allowing the expression of its internal sequence to be maintained. When the amount of lncRNA sequence contributed by TEs is normalized by the genomic size of each family, it becomes clear that some DNA and LTR families contributed more than expected by chance (Fig. 3c).

In the final part of this analysis we determined human lncRNAs that are conserved in NHPs, and used this information to determine which TE families contribute the most sequence to these conserved transcripts. This analysis revealed TE families that overlap most frequently 

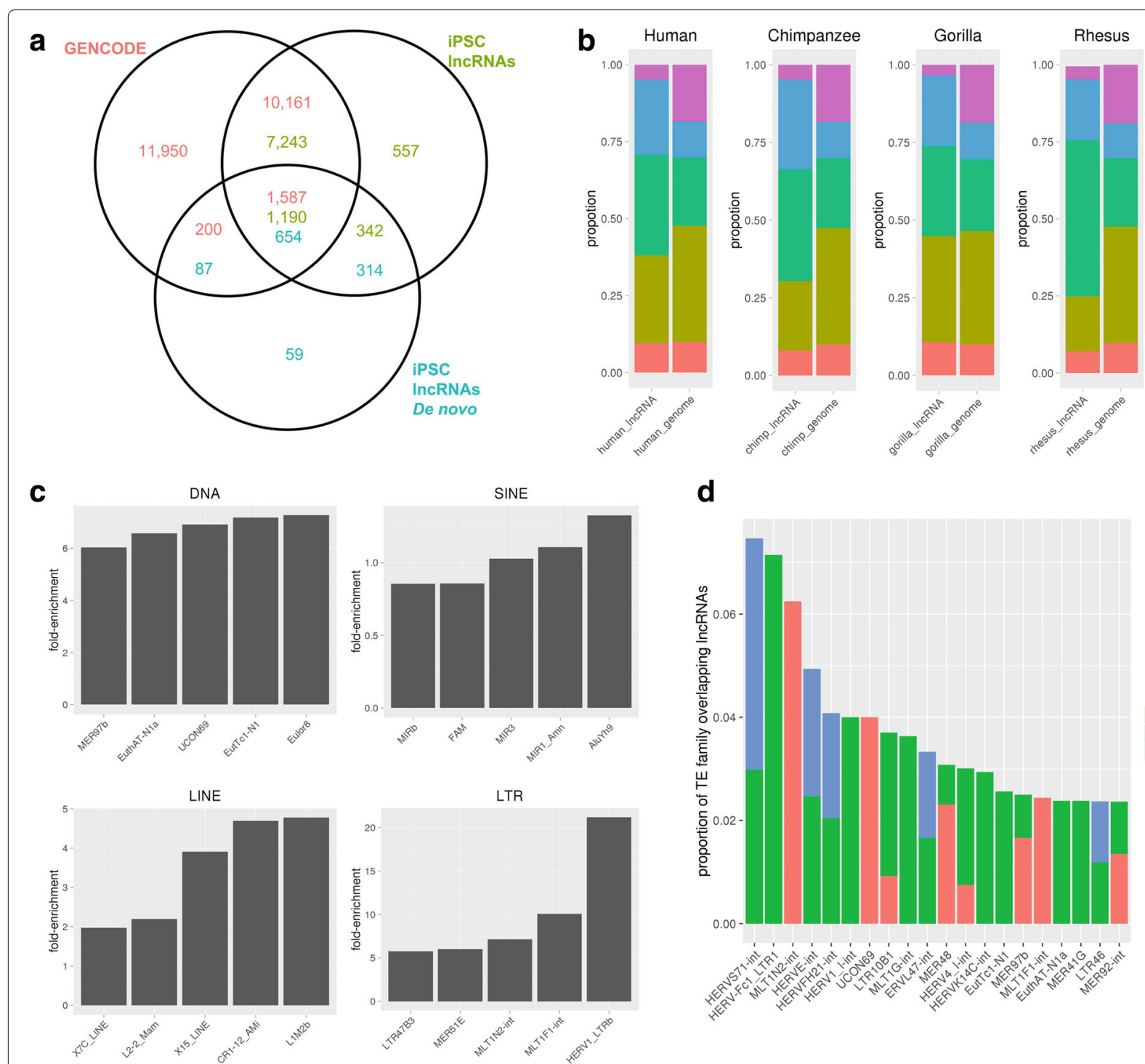

d

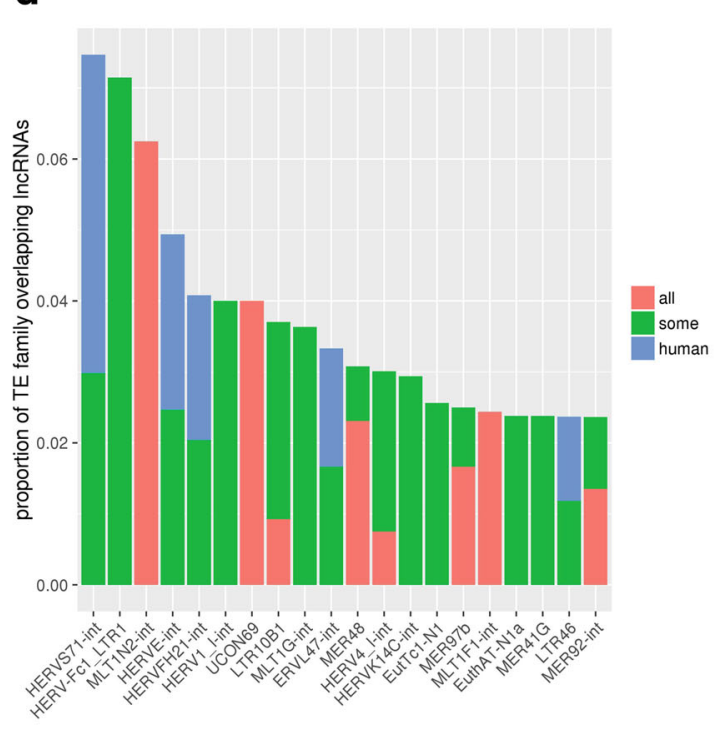

Fig. 3 a The intersection of 3 IncRNA annotations: iPSC with guide annotations, iPSC without guide, and GENCODE IncRNAs expressed in iPSCs. Over $90 \%$ of FEELnc human IncRNAs overlap with GENCODE IncRNAs. b The proportions in each primate IncRNA annotation compared to the genomic TE proportion. Only IncRNAs that overlap TEs are included in these proportions. c The proportion of human IncRNA sequence made up by TE families normalized by proportion of the genomic sequence made up by each family. The top 5 families from each of the 4 main classes are shown. $\mathbf{d}$ TEs that occur most frequently in human IPSC IncRNAs normalized by the size of each TE family. Only families with more than 10 members are shown. Red represents IncRNAs which are conserved in all 4 primate species. Green are those conserved in 1 or 2 other NHPs. Blue are human specific IncRNAs

Table 2 Conservation and TE contribution to human iPSC IncRNAs

\begin{tabular}{lllll}
\hline Lift human to & LiftOver & LiftOver, TEs & Expressed in target species & LiftOver, Expressed, TEs \\
\hline Chimpanzee & 7479 & $5175(69.19 \%)$ & $2981(39.86 \%)$ & $2103(28.12 \%)$ \\
Gorilla & 6709 & $4707(70.16 \%)$ & $2086(31.09 \%)$ & $1465(21.84 \%)$ \\
Rhesus & 6208 & $4550(73.29 \%)$ & $1527(24.60 \%)$ & $1351(21.77 \%)$ \\
\hline
\end{tabular}

Column 2: The number of human IncRNAs which lift to each NHP (out of 9332 human IncRNAs). Column 3: The number of lifted IncRNAs which overlap TEs in the target species. Column 4: Lifted human IncRNAs that are expressed in the target species. Column 5: Lifted and expressed IncRNAs that overlap TEs 
with human lncRNAs and how many of these transcripts are conserved in all, some, or none of the NHPs (Fig. 3d, Additional file 1: Figure S8). We identified several subfamilies of MLT1 that contributed large amounts of sequence to these conserved transcripts (Additional file 1: Figure S8), even when we took into account the genomic size of these subfamilies (Fig. 3d). For instance, two MLT1 subfamilies contribute the most to conserved lncRNA: MLT1N2 and MLT1F1. The MIRb family overlapped most frequently with human lncRNAs and is very well conserved in NHPs. While this TE family has been associated with non-coding variants of the CHRM2 gene [39], which is involved in neuron function, it is not widely recognized as contributing to lncRNAs.

\section{Discussion}

In this work, we utilized RNA-seq data from primate iPSCs to identify TEs producing potentially functional non-coding transcripts. We were able to characterize a number of TE families that are well conserved among human, chimpanzee, gorilla, and rhesus. In particular, we were able to identify a number of TE families with conserved expression (Fig. 2, Table 1). The conservation of TE expression across several primate species is an indicator of potential function and some of the TE families that we identified in this way, most notably HERVH $[13,14]$, have an already well-characterized biological function. Others, like MER53, are potentially interesting due to their conserved expression profile (Fig. 2c-d) and the fact that they are the source of a placental-specific family of miRNAs [34]. The family Tigger4a, from the DNA repeat class, also had high expression conservation when summed across all primate species (Fig. $2 \mathrm{~d}$ ) and highly significant $p$-value when tested for enrichment of expressed instances in conserved TEs between human and chimpanzee (Table 1). Tiggers are part of the mariner/Tc1 family originally discovered in drosophila [40], but not much is known about the potential function of Tiggers in human.

In this study, we also utilized the RNA-seq data to create iPSC-specific lncRNA annotations for these four primate species. Using these catalogues we identified TE families which contributed the most lncRNA sequence in human and non-human primates. We also noted TE families that most frequently occur in conserved lncRNA transcripts. Some of the TE families that appeared several times in our analysis are MLT1-type families (Figs. 2d, 3c-d, Additional file 1: Figure S7). Previous studies have shown that at least one of these families, MLT1J, harbors transcription factor binding sites for YY1 [38], which could explain why its expression has been maintained. We also noted that several Tigger families appear to have also contributed a significant amount of sequence to human lncRNAs (Additional file 1: Figure S7). Large-scale cDNA studies $[41,42]$ have identified several putative, Tigger-derived proteins, but based on these results we believe some members of the Tigger family may have also contributed important sequences to the primate IncRNA repertoire. These examples are just a few of the TE families that were highlighted in this study and potentially play important roles as non-coding transcripts in primate stem cells.

\section{Conclusions}

Our study focused on conserved expression of TE-derived lncRNAs, but many of the conserved TEs that were identified do not overlap lncRNAs. It is possible that TEs with conserved primate expression are being transcribed as by-products of an exapted enhancer or promoter, or as other transcripts like miRNAs. In a way, our list of potentially interesting TEs could be useful to explore the impact of TEs on other genomic functions. Several of the conserved TE families that were discussed here are not well characterized and would be good candidates for further experimental validation. In particular, experiments such as knockdowns and subcellular localization could be used to better determine their biological function.

\section{Methods}

\section{Generating transposable element annotations}

Using the genome builds hg19, panTro4, gorGor3, and rheMac3 for human, chimpanzee, gorilla, and rhesus, respectively, we generated TE annotations. TEs were annotated for each genome using RepeatMasker version 4.0.510 [28], RepBase Library version 20140131 [43], and the UCSC script extractNestedRepeats.pl [29]. We created our own TE annotation for this study to ensure consistency between assemblies. RepeatMasker was run using the following options: species - to identify the appropriate species, slow - to increase accuracy, and parallelize - to decrease runtime. After joining all the chromosome output files the results of RepeatMasker were passed to extractNestedRepeats.pl [29], which is a script available on the UCSC genome wiki page about the generation of UCSC repeat tracks. This is the method used to create the NestedRepeats track in the UCSC genome browser. extractNestedRepeats.pl uses the ID values given by RepeatMasker to join repeat instances that likely belong to the same insertion. In its original format the script only outputs repeats which can be merged, so we modified it to also output any repeats that did not require joining. This creates a complete track of repeat instances. The RepeatMasker output also includes information about the divergence of each TE from its family's consensus sequence. This is determined using a SmithWaterman alignment of each TE against its consensus, this score is averaged and transformed to a 0-1000 scale by extractNestedRepeats.pl for shading in the UCSC genome browser. In this study the consensus sequence is taken 
as an approximation of the ancestral sequence. Since TE sequence typically degrades over time we can estimate age from sequence divergence [32]. The age of whole TE families was determined by averaging the Smith-Waterman score of all family members.

\section{Identifying orthologous transposable elements}

We identified orthologous regions for human TEs in non-human primates (NHPs) using the UCSC LiftOver utility [44]. This program converts genome coordinates between genome assemblies. It uses a pairwise alignment of the genomes (chain files) generated by UCSC to convert given intervals between species. An important option in LiftOver is the -minMatch, or the minimum ratio of bases that must remap. $10 \%$ is the default minimum value on the UCSC genome browser when lifting between different species' genome builds. In order to confirm the option -minMatch 0.1 is appropriate we compared the lifted annotation with the RepeatMasker annotation in the target species at several cut off values. In particular, we examined HERVH annotations since we can infer the expected conservation based on the evolutionary history of its insertions. HERVH was lifted to each genome, and then verified to be annotated as HERVH by RepeatMasker in the target genome build. In all 3 primate species we found that at least $85 \%$ of the lifted HERVH are correctly, independently annotated as HERVH (Additional file 1: Table S2). This provided some evidence that a rate of $10 \%$ remapping is an appropriate value to accurately lift repeat intervals.

\section{Primate iPSC cell culture}

The Human iPS cell lines WT-33, ADRC-40 and WT126 were previously described [45], as was the method for generating iPSC cell lines for non-human primates [46]. Briefly, Fibroblasts from P. troglodytes (chimpanzees: PR00818), G. gorilla (gorilla: PR00053 and PR00075), and M. mulatta (rhesus) were from Coriell Cell Repositories (NJ). All fibroblasts were cultured in MEM (Invitrogen) supplemented with 10\% FBS (HyClone Laboratories). Retroviral vectors expressing OCT4 (also known as POU5F1), MYC, KLF4 and SOX2 human cDNAs from Yamanaka's group [47] were obtained from Addgene. Recombinant viruses were produced by transient transfection in 293T cells (ATCC - CRL-3216), as previously described [48]. Two days after infection, cells were plated on mitotically inactivated mouse embryonic fribroblasts (Chemicon) with human ES cell medium. after 2-4 weeks, iPSC cell colonies were picked manually and directly transferred to feeder-free conditions on matrigel-coated dishes (BD) using mTeSR1 (StemCell Technologies). Established iPS cell colonies were kept in feeder-free conditions indefinitely, and passed using mechanical dissociation. Embryoid-body-mediated differentiation in suspension was carried out for 10 days in the absence of growth factors.

iPSC clones continuously expressed pluripotency markers, retained undifferentiated morphology in culture, and maintained a normal karyotype. After embryoid body (EB)-mediated differentiation in vitro, clones contained tissue derivatives from the three embryonic germ layers and down-regulated expression of pluripotency markers.

\section{RNA-seq data generation}

Three human, 1 chimpanzee, 2 gorilla, and 1 rhesus cell lines were generated and sequenced. RNA was isolated with miRNeasy Mini Kit (Qiagen) and 500ng of RNA were used to prepare libraries using Illumina TruSeq Stranded Total RNA Sample preparation kit following manufacturers directions. Quality control was performed using Bioanalyzer and samples were sequenced on Illumina HiSeq2000, 100bp paired-end reads. Raw reads were trimmed (quality: phred33 $\geq 30$ and length $n \geq 32$ ), adapters were removed (using Trimmomatic V.0.32 [49]) and reads were aligned to the hg19 human reference (Tophat v.2.0.10 [50] and bowtie v.2.1.0 [51]). We received samples in Trizol and then cleaned up the RNA. All the sequencing was done on Illumina HiSeq2000 100bp paired-end and indexed at 4-fold.

Gene expression clustering was done using protein coding genes that are orthologous between human and all 3 non-human primates. Human and non-human primate reads were mapped to hg19 and expression of protein coding genes was determined using HTSeq-count [52] and DESeq2 [53]. Clustering of top 1000 genes is shown in Additional file 1: Figure S9. PCA analysis of all orthologous protein coding genes was also performed (Additional file 1: Figure S10).

\section{Determining transposable element expression}

Expression analysis was carried out on each species by first counting the coverage of RNA-seq reads over each TE. This step was performed using coverageBed from the BEDTools suite [54]. The counts were then normalized by library size and TE length using the method reads per kilobase of transcripts per million mapped reads (RPKM) [55]. Next, in the case of human and gorilla where we have biological replicates, we average the RPKM values over the replicates. Expressed TEs are defined as those with an RPKM of 1 or greater. The expression analysis was only conducted on TEs which do not directly overlap coding regions. In identifying TEs with conserved expression between species we only counted TEs which LiftOver and are annotated as a TE in the target species.

\section{Statistical analysis}

We performed statistical analysis on the conserved sets of TE families. We utilized a hypergeometric test to 
determine whether the set of conserved TE sequences is enriched for being expressed in primate iPSCs. We wanted to know if there is an association between sequence conservation and expression. In a pairwise comparison of primate species this test gives us the probability of seeing $j$ or more expression conserved TEs from a set of $i$ TEs that are expressed in one of the species. Generally, the hypergeometric distribution is a discrete probability distribution describing the number of successful draws from a finite population without replacement.

We utilized a number of filtering techniques in order to ensure that our TE lists contain genuinely interesting families. To this end we removed very small TE families and simple repeats. Many of these very small families exhibited significant $p$-values when we examined for enrichment in conservation of expression between primates, but this is likely due to bias in the statistical test. Additionally these small families are much less likely to give rise to lncRNA transcripts, which is another reason why these particular types of TEs are not interesting in the context of this study. Moreover, simple repeats and low complexity regions frequently occur in high GC regions, which may affect their detection [19].

\section{Creating IncRNA catalogs}

lncRNA annotations were generated for all four species using a combination of our own filtering techniques and a pipeline available online for annotating lncRNAs called FEELnc (Additional file 1: Figure S5) [37]. The first step of this analysis was to assemble the RNA-seq transcriptome for each species. This was done using Cufflinks [50] with default parameters and ensembl gene annotations as a guide. The guide annotations were obtained from UCSC for the genome builds hg19, panTro4, gorGor3, and rheMac3 for human, chimpanzee, gorilla, and rhesus, respectively. In the case of human and gorilla, for which we have biological replicate data, we also used Cuffmerge to merge the Cufflinks transcriptomes. Cuffmerge was also run with default parameters.

After we produced the iPSC transcriptome for each primate species we used FEELnc to filter out any transcripts that are not long non-coding. We generated our own filter file to remove any known transcripts other than IncRNAs. This includes protein coding genes, pseudogenes, and tRNAs among others (See Additional file 1: Table S7 for full biotypes list). This filtering step also removes mono-exonic transcripts. While there do exist some mono-exonic lncRNAs there are very few, and they are difficult to evaluate as true lncRNAs [24].

The next step of the pipeline removes transcripts with protein coding potential. To do this we used a version of the FEELnc pipeline which utilizes CPAT [13]. The optimal cutoff value for coding potential is calculated by
CPAT using a training set of coding genes and intergenic regions. CPAT uses a 10 fold cross-validation on the training data to maximize sensitivity and specificity. Any transcripts with high protein coding potential are removed from our catalogues.

The method for annotating lncRNAs was evaluated by comparing our own human annotation against the GENCODE IncRNA annotation (version 19) [25]. After determining that the level of lncRNA detection was acceptable in human we used the same method to annotate lncRNAs in the non-human primates.

The lncRNA catalogues resulting from this pipeline had low numbers of transcripts annotated in NHP compared to human. We speculated that this was due to the fact that non-human primate genomes have poorer gene annotations compared to human. To test this we reran the pipeline without passing guide annotations to Cufflinks.

\section{Identifying conserved transcripts}

After creating the lncRNA catalogues we used LiftOver to evaluate orthologous regions between the primate species. Based on the validation from TE LiftOver we again used 0.1 for the minimum ratio of bases that must remap. The conservation of lncRNAs was done based on our human annotation. We lifted lncRNAs from human to each of the 3 NHPs. We then performed expression analysis in non-human primates on the LiftOver lncRNAs to determine which are also expressed in NHP. RPKM values were calculated for each transcript using the Bioconductor package Rsubread. Reads were counted using featureCounts(), and normalized using rpkm() [56]. Expressed transcripts are defined as those with 1 RPKM or greater. For species with biological replicates this cutoff was required in all replicates to be deemed expressed.

\section{Annotating IncRNAs with TEs}

After creating lncRNA catalogues for human, chimpanzee, gorilla, and rhesus we annotated the transcripts with TEs. This analysis uses two TE annotation methods. The first is a simple intersection between lncRNA exons and TEs. This was done using intersectBed from the BEDTools Suite [54]. We used the parameters -wao to output all intersection information (which features overlap and by how many base pairs), and -f 0.1 to require that at least $10 \%$ of an exon must overlap a TE for the intersection to be counted. An intersection of all TEs with our lncRNAs allowed us the look at which TEs contribute the most lncRNA sequence and examine the enrichment of specific families' sequence contribution to lncRNAs. The second method of TE annotation is to label each lncRNA that overlaps TEs with a single TE (the sole element that overlaps, or the one that overlaps the most). This allows us to classify lncRNAs by TE family and evaluate which TE families occur most frequently. 


\section{Additional files}

Additional file 1: Supplementary Tables and Figures. (PDF 769 kb)

Additional file 2: Table of values for figures 1 and 2 and table 1 . (TXT $1220 \mathrm{~kb}$ )

Additional file 3: Table of coordinates for table 1. (TXT 869 kb)

\section{Abbreviations}

ERV: Endogenous retrovirus; HERVH: Human endogenous retrovirus subfamily h; hESC: Human embryonic stem cells; iPSCs: Induced pluripotent stem cells; IncRNA: Long non-coding RNA; NHP: Non-human primate; TE: Transposable element

\section{Acknowledgments}

Data analyses were enabled by compute and storage resources provided by Compute Canada and Calcul Quebec. We are grateful to Thomas Derrien for his assistance with his IncRNA pipeline, FEELnc.

\section{Funding}

This work was supported by grants from the Canadian Institute for Health Research (MOP-115090, EP1-120608, EP2-120609 to GB and TP) and funds from the NIH (R01 MH095741, U01 MH106882 to FHG). FHG would also like to acknowledge funding from The G. Harold and Leila Y. Mathers Charitable Foundation, The Leona M. and Harry B. Helmsley Charitable Trust (grant \#2012-PG-MED00), The Engman Foundation, Annette C. Merle-Smith, Takeda Pharmaceutical International, Paul G. Allen Family Foundation, Glenn Center for Aging Research at the Salk Institute and JPB Foundation. GB is supported by the Fonds de Recherche Sante Quebec (FRSQ-25348). The funding agencies had no role in the design, analysis, interpretation of the data or writing of the manuscript.

\section{Availability of data and materials}

The RNA-seg data is available through the IHEC Data Portal [57]

Primates: http://epigenomesportal.ca/ihec/lHECDP00000039

Humans: http://epigenomesportal.ca/ihec/IHECDP00000040

Raw datasets are also available at the European Genome-phenome Archive under the accession ID EGAD00001001276.

\section{Authors' contributions}

LR contributed to the design, performed bioinformatic analyses, and wrote the manuscript. CM generated the iPSC cell cultures and performed RNA extraction. S-HC prepared the samples for RNA sequencing. MC performed initial processing of the RNA-seq data. SB, TK, TP, and FHG provided support for sequencing and data generation. GB designed the study, assisted in the interpretation of results and wrote the manuscript. All authors have read and approved the final version of the manuscript.

\section{Competing interests}

The authors declare that they have no competing interests.

\section{Consent for publication}

Not applicable.

\section{Ethics approval and consent to participate}

The use of non-human primate samples was approved by the US Fish and Wildlife Service, under the permit MA206206. Protocols describing the use of iPS and human ES cells were previously approved by the University of California, San Diego (UCSD), the Salk Institute Institutional Review Board and the Embryonic Stem Cell Research Oversight Committee.

\footnotetext{
Author details

${ }^{1}$ Department of Human Genetics, McGill University, 1205 Dr Penfield Avenue, H3A 1B1 Montreal, Canada. ${ }^{2}$ Lab of Genetics, Salk Institute for Biological Studies, 10010 N Torrey Pines Rd, CA 92037 La Jolla, USA. ${ }^{3}$ McGill University and Genome Quebec Innovation Centre, 740 Dr Penfield Avenue, H3A 1A4 Montreal, Canada
}

Received: 1 September 2016 Accepted: 7 February 2017

Published online: 28 February 2017

\section{References}

1. The ENCODE Project Consortium. An integrated encyclopedia of dna elements in the human genome. Nature. 2012;489(7414):57-74.

2. Dinger ME, Amaral PP, Mercer TR, Mattick JS. Pervasive transcription of the eukaryotic genome: functional indices and conceptual implications. Brief Funct Genomic Proteomic. 2009;8(6):407-23.

3. Kim TK, Hemberg M, Gray JM, Costa AM, Bear DM, Wu J, Harmin DA, Laptewicz M, Barbara-Haley K, Kuersten S, Markenscoff-Papadimitriou E, Kuhl D, Bito H, Worley PF, Kreiman G, Greenberg ME. Widespread transcription at neuronal activity-regulated enhancers. Nature. 2010;465(7295):182-7.

4. Nagano T, Mitchell JA, Sanz LA, Pauler FM, Ferguson-Smith AC, Feil R, Fraser $P$. The air noncoding rna epigenetically silences transcription by targeting g9a to chromatin. Science. 2008;322(5908):1717-20.

5. Huarte M, Guttman M, Feldser D, Garber M, Koziol MJ, Kenzelmann-Broz D, Khalil AM, Zuk O, Amit I, Rabani M, Attardi LD, Regev A, Lander ES, Jacks T, Rinn JL. A large intergenic noncoding rna induced by p53 mediates global gene repression in the p53 response. Cell. 2010;142(3):409-19.

6. Lander ES, Linton LM, Birren B, Nusbaum C, Zody MC, Baldwin J, Devon K, Dewar K, Doyle M, FitzHugh W, Funke R, Gage D, Harris K, Heaford A, Howland J, Kann L, Lehoczky J, LeVine R, McEwan P, McKernan K, Meldrim J, Mesirov JP, Miranda C, Morris W, Naylor J, Raymond C, Rosetti M, Santos R, Sheridan A, Sougnez C, Stange-Thomann N, Stojanovic N, Subramanian A, Wyman D, Rogers J, Sulston J, Ainscough R, Beck S, Bentley D, Burton J, Clee C, Carter N, Coulson A, Deadman R, Deloukas P, Dunham A, Dunham I, Durbin R, French L, Grafham D, Gregory S, Hubbard T, Humphray S, Hunt A, Jones M, Lloyd C, McMurray A, Matthews L, Mercer S, Milne S, Mullikin JC, Mungall A, Plumb R, Ross M, Shownkeen R, Sims S, Waterston RH, Wilson RK, Hillier LW, McPherson JD, Marra MA, Mardis ER, Fulton LA, Chinwalla AT, Pepin KH, Gish WR, Chissoe SL, Wendl MC, Delehaunty KD, Miner TL, Delehaunty A, Kramer JB, Cook LL, Fulton RS, Johnson DL, Minx PJ, Clifton SW, Hawkins T, Branscomb E, Predki P, Richardson P, Wenning S, Slezak T, Doggett N, Cheng JF, Olsen A, Lucas S, Elkin C, Uberbacher E, Frazier M, et al. Initial sequencing and analysis of the human genome. Nature. 2001:409(6822):860-921.

7. Kapusta A, Kronenberg Z, Lynch VJ, Zhuo X, Ramsay L, Bourque G, Yandell M, Feschotte C. Transposable elements are major contributors to the origin, diversification, and regulation of vertebrate long noncoding rnas. PLoS Genet. 2013;9(4):1003470.

8. Johnson R, Guigo R. The ridl hypothesis: transposable elements as functional domains of long noncoding rnas. Rna. 2014;20(7):959-76.

9. Loewer S, Cabili MN, Guttman M, Loh YH, Thomas K, Park IH, Garber M, Curran M, Onder T, Agarwal S, Manos PD, Datta S, Lander ES, Schlaeger TM, Daley GQ, Rinn JL. Large intergenic non-coding rna-ror modulates reprogramming of human induced pluripotent stem cells. Nat Genet. 2010;42(12):1113-7.

10. Jern P, Sperber GO, Blomberg J. Definition and variation of human endogenous retrovirus h. Virology. 2004;327(1):93-110.

11. Kelley D, Rinn J. Transposable elements reveal a stem cell-specific class of long noncoding rnas. Genome Biol. 2012;13(11):107.

12. Santoni FA, Guerra J, Luban J. Herv-h rna is abundant in human embryonic stem cells and a precise marker for pluripotency. Retrovirology. 2012;9:111

13. Wang J, Xie G, Singh M, Ghanbarian AT, Rasko T, Szvetnik A, Cai H, Besser D, Prigione A, Fuchs NV, Schumann GG, Chen W, Lorincz MC, Ivics Z, Hurst LD, Izsvak Z. Primate-specific endogenous retrovirus-driven transcription defines naive-like stem cells. Nature. 2014;516(7531):405-9.

14. LuX, Sachs F, Ramsay L, Jacques PE, Goke J, Bourque G, Ng HH. The retrovirus hervh is a long noncoding rna required for human embryonic stem cell identity. Nat Struct Mol Biol. 2014;21(4):423-5.

15. Guttman M, Donaghey J, Carey BW, Garber M, Grenier JK, Munson G, Young G, Lucas AB, Ach R, Bruhn L, Yang X, Amit I, Meissner A, Regev A, Rinn JL, Root DE, Lander ES. lincrnas act in the circuitry controlling pluripotency and differentiation. Nature. 2011;477(7364):295-300.

16. Wicker T, Sabot F, Hua-Van A, Bennetzen JL, Capy P, Chalhoub B, Flavell A, Leroy P, Morgante M, Panaud O, Paux E, SanMiguel P, Schulman $\mathrm{AH}$. A unified classification system for eukaryotic transposable elements. Nat Rev Genet. 2007;8(12):973-82.

17. Cowley M, Oakey RJ. Transposable elements re-wire and fine-tune the transcriptome. PLoS Genet. 2013;9(1):1003234 
18. Kunarso G, Chia NY, Jeyakani J, Hwang C, Lu X, Chan YS, Ng HH, Bourque $\mathrm{G}$. Transposable elements have rewired the core regulatory network of human embryonic stem cells. Nat Genet. 2010;42(7):631-4

19. Jacques $P E$, Jeyakani J, Bourque $G$. The majority of primate-specific regulatory sequences are derived from transposable elements. PLoS Genet. 2013;9(5):1003504

20. Chow JC, Yen Z, Ziesche SM, Brown CJ. Silencing of the mammalian $x$ chromosome. Annu Rev Genomics Hum Genet. 2005;6:69-92.

21. Boffelli D, McAuliffe J, Ovcharenko D, Lewis KD, Ovcharenko I, Pachter L, Rubin EM. Phylogenetic shadowing of primate sequences to find functional regions of the human genome. Science. 2003;299(5611): $1391-4$.

22. Lowe CB, Bejerano G, Haussler D. Thousands of human mobile element fragments undergo strong purifying selection near developmental genes. Proc Natl Acad Sci USA. 2007:104(19):8005-10.

23. Necsulea A, Soumillon M, Warnefors M, Liechti A, Daish T, Zeller U, Baker JC, Grutzner F, Kaessmann H. The evolution of Incrna repertoires and expression patterns in tetrapods. Nature. 2014;505(7485):635-40.

24. Cabili MN, Trapnell C, Goff L, Koziol M, Tazon-Vega B, Regev A, Rinn JL. Integrative annotation of human large intergenic noncoding rnas reveals global properties and specific subclasses. Genes Dev. 2011;25(18): 1915-27.

25. Derrien T, Johnson R, Bussotti G, Tanzer A, Djebali S, Tilgner H, Guernec G, Martin D, Merkel A, Knowles DG, Lagarde J, Veeravalli L, Ruan X, Ruan Y, Lassmann T, Carninci P, Brown JB, Lipovich L, Gonzalez JM, Thomas M, Davis CA, Shiekhattar R, Gingeras TR, Hubbard TJ, Notredame C, Harrow J, Guigo R. The gencode v7 catalog of human long noncoding rnas: analysis of their gene structure, evolution, and expression. Genome Res. 2012;22(9):1775-89.

26. Zheng LL, Li JH, Wu J, Sun WJ, Liu S, Wang ZL, Zhou H, Yang JH, Qu LH. deepbase v2.0: identification, expression, evolution and function of small rnas, Incrnas and circular rnas from deep-sequencing data. Nucleic Acids Res. 2016;44(D1):196-202

27. Chen J, Shishkin AA, Zhu X, Kadri S, Maza I, Guttman M, Hanna JH, Regev A, Garber M. Evolutionary analysis across mammals reveals distinct classes of long non-coding rnas. Genome Biol. 2016 Feb 2;17:19.

28. Smit A, Hubley R, Green P. RepeatMasker Open-4.0. http://www. repeatmasker.org

29. Karolchik D, Barber GP, Casper J, Clawson H, Cline MS, Diekhans M, Dreszer TR, Fujita PA, Guruvadoo L, Haeussler M, Harte RA, Heitner S, Hinrichs AS, Learned K, Lee BT, Li CH, Raney BJ, Rhead B, Rosenbloom KR, Sloan CA, Speir ML, Zweig AS, Haussler D, Kuhn RM, Kent WJ. The ucsc genome browser database: 2014 update. Nucleic Acids Res. 2014;42(Database issue):764-0.

30. Goodchild NL, Freeman JD, Mager DL. Spliced herv-h endogenous retroviral sequences in human genomic dna: evidence for amplification via retrotransposition. Virology. 1995;206(1):164-73.

31. Mager DL, Freeman JD. Herv-h endogenous retroviruses: presence in the new world branch but amplification in the old world primate lineage. Virology. 1995;213(2):395-404.

32. Bourque $G$, Leong $B$, Vega VB, Chen $X$, Lee $Y L$, Srinivasan $K G$, Chew JL, Ruan Y, Wei $\mathrm{CL}, \mathrm{Ng} \mathrm{HH}$, Liu ET. Evolution of the mammalian transcription factor binding repertoire via transposable elements. Genome Res. 2008;18(11):1752-62.

33. Bennett EA, Keller H, Mills RE, Schmidt S, Moran JV, Weichenrieder O, Devine SE. Active alu retrotransposons in the human genome. Genome Res. 2008;18(12):1875-83.

34. Yuan Z, Sun X, Jiang D, Ding Y, Lu Z, Gong L, Liu H, Xie J. Origin and evolution of a placental-specific microrna family in the human genome. BMC Evol Biol. 2010;10:346.

35. Morin RD, O'Connor MD, Griffith M, Kuchenbauer F, Delaney A, Prabhu AL, Zhao Y, McDonald H, Zheng T, Hirst M, Eaves CJ, Marra MA. Application of massively parallel sequencing to microrna profiling and discovery in human embryonic stem cells. Genome Research. 2008;18(4): 610-21.

36. Klawitter S, Fuchs NV, Upton KR, Munoz-Lopez M, Shukla R, Wang J, Garcia-Canadas M, Lopez-Ruiz C, Gerhardt DJ, Sebe A, Grabundzija I, Merkert S, Gerdes P, Pulgarin JA, Bock A, Held U, Witthuhn A, Haase A, Sarkadi B, Lower J, Wolvetang EJ, Martin U, Ivics Z, Izsvak Z, Garcia-Perez JL, Faulkner GJ, Schumann GG. Reprogramming triggers endogenous $\mid 1$ and alu retrotransposition in human induced pluripotent stem cells. Nat Commun. 2016;7:10286.
37. Derrien T. FlExible Extraction of LncRNA. https://github.com/tderrien/ FEELnc. Accessed 06 Aug 2015.

38. Schwalie PC, Ward MC, Cain CE, Faure AJ, Gilad Y, Odom DT, Flicek P. Co-binding by yy 1 identifies the transcriptionally active, highly conserved set of ctcf-bound regions in primate genomes. Genome Biol. 2013;14(12):148.

39. Gosso FM, de Geus EJ, Polderman TJ, Boomsma DI, Posthuma D, Heutink P. Exploring the functional role of the chrm2 gene in human cognition: results from a dense genotyping and brain expression study. BMC Med Genet. 2007;8:66.

40. Smit AF, Riggs AD. Tiggers and dna transposon fossils in the human genome. Proc Natl Acad Sci USA. 1996;93(4):1443-8.

41. Gerhard DS, Wagner L, Feingold EA, Shenmen CM, Grouse LH, Schuler G, Klein SL, Old S, Rasooly R, Good P, Guyer M, Peck AM, Derge JG, Lipman D, Collins FS, Jang W, Sherry S, Feolo M, Misquitta L, Lee E, Rotmistrovsky K, Greenhut SF, Schaefer CF, Buetow K, Bonner TI, Haussler D, Kent J, Kiekhaus M, Furey T, Brent M, Prange C, Schreiber K, Shapiro N, Bhat NK, Hopkins RF, Hsie F, Driscoll T, Soares MB, Casavant TL, Scheetz TE, Brown-stein MJ, Usdin TB, Toshiyuki S, Carninci P, Piao Y, Dudekula DB, Ko MS, Kawakami K, Suzuki Y, Sugano S, Gruber CE, Smith MR, Simmons B, Moore T, Waterman R, Johnson SL, Ruan Y, Wei $\mathrm{CL}$, Mathavan S, Gunaratne PH, Wu J, Garcia AM, Hulyk SW, Fuh E, Yuan Y, Sneed A, Kowis C, Hodgson A, Muzny DM, McPherson J, Gibbs RA, Fahey J, Helton E, Ketteman M, Madan A, Rodrigues S, Sanchez A, Whiting M, Madari A, Young AC, Wetherby KD, Granite SJ, Kwong PN, Brinkley CP, Pearson RL, Bouffard GG, Blakesly RW, Green ED, Dickson MC, Rodriguez AC, Grimwood J, Schmutz J, Myers RM, Butterfield YS, Griffith M, Griffith OL, Krzywinski MI, Liao N, Morin R, Palmquist D, et al. The status, quality, and expansion of the nih full-length cdna project: the mammalian gene collection (mgc). Genome Res. 2004;14(10b):2121-7.

42. Volff JN. Turning junk into gold: domestication of transposable elements and the creation of new genes in eukaryotes. Bioessays. 2006;28(9): 913-22.

43. Jurka J, Kapitonov W, Pavlicek A, Klonowski P, Kohany O, Walichiewicz J. Repbase update, a database of eukaryotic repetitive elements. Cytogenet Genome Res. 2005;110(1-4):462-7.

44. Kent WJ, Sugnet CW, Furey TS, Roskin KM, Pringle TH, Zahler AM, Haussler D. The human genome browser at ucsc. Genome Res. 2002;12(6):996-1006.

45. Marchetto MC, Carromeu C, Acab A, Yu D, Yeo GW, Mu Y, Chen G, Gage FH, Muotri AR. A model for neural development and treatment of rett syndrome using human induced pluripotent stem cells. Cell. 2010;143(4):527-39.

46. Marchetto MCN, Narvaiza I, Denli AM, Benner C, Lazzarini TA, Nathanson JL, Paquola ACM, Desai KN, Herai RH, Weitzman MD, Yeo GW, Muotri AR, Gage FH. Differential line-1 regulation in pluripotent stem cells of humans and other great apes. Nature. 2013;503(7477):525-9.

47. Takahashi K, Yamanaka S. Induction of pluripotent stem cells from mouse embryonic and adult fibroblast cultures by defined factors. Cell. 2006;126(4):663-76.

48. Muotri AR, Nakashima K, Toni N, Sandler VM, Gage FH. Development of functional human embryonic stem cell-derived neurons in mouse brain. Proc Natl Acad Sci USA. 2005;102(51):18644-8.

49. Bolger AM, Lohse M, Usadel B. Trimmomatic: a flexible trimmer for illumina sequence data. Bioinformatics. 2014;30(15):2114-0.

50. Trapnell C, Williams BA, Pertea G, Mortazavi A, Kwan G, van Baren MJ, Salzberg SL, Wold BJ, Pachter L. Transcript assembly and quantification by rna-seq reveals unannotated transcripts and isoform switching during cell differentiation. Nat Biotechnol. 2010;28(5):511-5.

51. Langmead B, Trapnell C, Pop M, Salzberg SL. Ultrafast and memory-efficient alignment of short dna sequences to the human genome. Genome Biol. 2009;10(3):25.

52. Anders S, Pyl PT, Huber W. Htseq-a python framework to work with high-throughput sequencing data. Bioinformatics. 2015;31(2):166-9.

53. Love MI, Huber W, Anders S. Moderated estimation of fold change and dispersion for rna-seq data with deseq2. Genome Biol. 2014;15(12):550.

54. Quinlan AR, Hall IM. Bedtools: a flexible suite of utilities for comparing genomic features. Bioinformatics. 2010;26(6):841-2.

55. Mortazavi A, Williams BA, McCue K, Schaeffer L, Wold B. Mapping and quantifying mammalian transcriptomes by rna-seq. Nat Methods. 2008;5(7):621-8. 
56. Liao Y, Smyth GK, Shi W. The subread aligner: fast, accurate and scalable read mapping by seed-and-vote. Nucleic Acids Res. 2013;41(10):108.

57. Bujold D, Morais DA, Gauthier C, Cote C, Caron M, Kwan T, Chen KC, Laperle J, Markovits AN, Pastinen T, Caron B, Veilleux A, Jacques PE, Bourque G. The International Human Epigenome Consortium Data Portal. Cell Syst. 2016;3(5):496-9.

Submit your next manuscript to BioMed Central and we will help you at every step:

- We accept pre-submission inquiries

- Our selector tool helps you to find the most relevant journal

- We provide round the clock customer support

- Convenient online submission

- Thorough peer review

- Inclusion in PubMed and all major indexing services

- Maximum visibility for your research

Submit your manuscript at www.biomedcentral.com/submit 